\title{
Phosphorylation of Ser6 in hnRNPA1 by S6K2 regulates glucose metabolism and cell growth in colorectal cancer
}

\author{
YAN SUN ${ }^{1}$, MAN LUO $^{1}$, GUILIN CHANG $^{1}$, WEIYING REN ${ }^{1}$, KEFEN WU $^{1}$, \\ XI LI ${ }^{1}$, JIPING SHEN ${ }^{1}$, XIAOPING ZHAO ${ }^{2}$ and YU HU ${ }^{1}$ \\ ${ }^{1}$ Department of Geriatrics, Zhongshan Hospital, Fudan University, Shanghai 200032; ${ }^{2}$ Department of Nuclear Medicine, \\ Ren Ji Hospital, School of Medicine, Shanghai Jiao Tong University, Shanghai 200127, P.R. China
}

Received January 15, 2016; Accepted July 27, 2017

DOI: $10.3892 / \mathrm{ol} .2017 .7085$

\begin{abstract}
Abnormal glucose metabolism is critical in colorectal cancer (CRC) development. Expression of the pyruvate kinase (PK) M2 isoform, rather than the PKM1 isoform, serves important functions in reprogramming the glucose metabolism of cancer cells. Preferential expression of PKM2 is primarily driven by alternative splicing, which is coordinated by a group of splicing factors including heterogeneous nuclear ribonucleoprotein (hnRNP)A1, hnRNPA2 and RNA binding motif containing. However, the underlying molecular mechanisms associated with cancer cell expression of PKM2, instead of PKM1, remain unknown. The mRNA levels of PKM isoform and glucose metabolism were analyzed in CRC cells. The results of the present study indicated that S6 kinase 2 (S6K2) promotes glycolysis and growth of CRC cells by regulating alternative splicing of the PKM gene. In addition, chromatin immunoprecipitation assay indicated that S6K2 phosphorylation of $\mathrm{Ser}^{6}$ of hnRNPA1 facilitated hnRNPA1 binding to the splicing site of the PKM gene. As a result, cancer cells preferentially expressed the PKM2 isoform, instead of the PKM1 isoform. Furthermore, Cox regression analysis demonstrated that the phosphorylation of $\operatorname{Ser}^{6}$ of hnRNPA1 was a predictor of poor prognosis for patients with CRC. Therefore, the results of the present study revealed that the phosphorylation of $\operatorname{Ser}^{6}$ in hnRNPA1 by S6K2 was a novel mechanism underlying glucose metabolic reprogramming, and suggested that S6K2 is a potential therapeutic target for CRC treatment.
\end{abstract}

Correspondence to: Dr Xiaoping Zhao, Department of Nuclear Medicine, Ren Ji Hospital, School of Medicine, Shanghai Jiao Tong University, 1630 Dongfang Road, Shanghai 200127, P.R. China E-mail: zxp0856@sina.com

Dr Yu Hu, Department of Geriatrics, Zhongshan Hospital, Fudan University, 180 Fenglin Road, Shanghai 200032, P.R. China E-mail: hu.yu@zs-hospital.sh.cn

Key words: alternative splicing, colorectal cancer, glucose metabolism, heterogeneous nuclear ribonucleoprotein, pyruvate kinase

\section{Introduction}

Colorectal cancer (CRC) is the third leading cause of all tumor-associated mortalities and resulted in 693,900 in 2012 worldwide in 2012, accounting for $8 \%$ of all cancer-associated mortalities (1). Although the prognosis of CRC has improved due to the development of surgical procedures and therapeutic agents, survival is rarely longer than three years (2). Consequently, the identification of novel diagnostic markers and the elucidation of the underlying molecular mechanisms of tumorigenesis of CRC are required.

Reprogramming metabolic pathways is the key characteristic of cancer cells (3). A number of types of cancer cell exhibit increased glucose consumption and lactate production independent of oxygen availability, a phenomenon termed the Warburg effect (4). Pyruvate kinase (PK), one of the rate-limiting enzymes of glycolysis, serves functions in modulating glucose usage and metabolic flux in adaption to different stresses during tumorigenesis (5). In mammals, four isoforms of pyruvate kinase (PKL, PKR, PKM1 and PKM2) have been identified. PKL and PKR are expressed in the liver and red blood cells, respectively (6). PKM1 is expressed in adult tissue, whereas PKM2 is expressed in embryonic tissue and tumors. PKM1 and PKM2 are products of alternative splicing of PKM gene, which contain a mutually exclusive exon (6). Exon 9 is specific for PKM1 and exon 10 exists in PKM2 (7). PKM1 is the constitutively active isoform, which preferentially diverts glucose into the citric acid cycle to generate adenosine triphosphate. However, PKM2 exhibits relatively low pyruvate kinase activity compared with PKM1, which causes glucose to be metabolized by glycolysis, rather than by oxidative phosphorylation. Furthermore, PKM2 may function as a protein kinase and has been demonstrated to be involved in multiple signaling pathways $(8,9)$. Therefore, PKM2 exhibits metabolic advantages in cancer cells and participates in oncogenic signaling networks. As a result, PKM2 is required for the rapid growth of cancer cells and tumorigenesis (10).

During the development of cancer, PKM gene expression is transformed from PKM1 to PKM2, which is controlled by a group of splicing factors. Three heterogeneous nuclear ribonucleoproteins (hnRNPs), including hnRNPA1, hnRNPA2 and PTBP1, have been identified to exclude exon 9 in the PKM transcript, which results in the expression of the PKM2 
isoform rather than the PKM1 isoform $(7,11)$. It has been previously demonstrated that microRNA (miR-)124 causes a switch in PKM gene expression from PKM2 to PKM1, by targeting polypyrimidine tract-binding protein (PTB) and hnRNPA2, which subsequently transforms glucose flux from glycolysis to oxidative phosphorylation (12). As a result, expressing miR-124 inhibits the growth of CRC cells by modulating glucose metabolism (12).

S6 kinase (S6K)1 and S6K2 are members of the AGC kinase super-family (13). The mechanistic target of rapamycin/S6K signaling pathway has been identified to be involved in a number of types of disease, including cancer and metabolic syndromes (14). The principal focus has been placed on S6K1 and S6K2 has been neglected due to their similarities in structure (15). However, a previous study indicated that these two isoforms have distinct biological functions (15). The overexpression of S6K2 in tumor tissues is more common, compared with that of S6K1 (16,17). Furthermore, S6K2, rather than S6K1, mediates pro-survival signaling of cancer cells (18). S6K2 phosphorylates hnRNPA1 on Ser4/6 sites, which promotes the translation of B-cell lymphoma-extra-large (BCL-XL) and X-linked inhibitor of apoptosis (XIAP), and exhibits pro-survival effects on cells (19). However, the underlying molecular mechanism of S6K2 in tumorigenesis remains unknown.

In the present study, the results indicated that $\mathrm{S} 6 \mathrm{~K} 2 \mathrm{regu}-$ lated glucose metabolism by modulating alternative splicing of the PKM gene. The mRNA ratio of PKM2/PKM1 was markedly increased by S6K2 in a hnRNPA1-dependent manner. As a result, glycolysis was induced by S6K2. Phosphorylation of $\mathrm{Ser}^{6}$, rather than $\mathrm{Ser}^{4}$, in hnRNPA1 by S6K2 is required to increase the PKM2 isoform and induce glycolysis in CRC cells. Notably, the level of $\operatorname{Ser}^{6}$ phosphorylation of hnRNPA1 predicted poor prognosis for patients with CRC. Therefore, the present study identified that S6K2-mediated regulation of glucose metabolism is involved in the development of CRC. $\mathrm{S} 6 \mathrm{~K} 2$ represents a novel metabolic target for CRC treatment.

\section{Materials and methods}

Cell culture and reagents. HCT116 cells were cells were purchased from the American Type Culture Collection (Manassas, VA, USA) and cultured in Dulbecco's modified Eagle's medium (DMEM; Invitrogen; Thermo Fisher Scientific, Inc., Waltham, MA, USA) supplemented with $10 \%$ heat-inactivated fetal bovine serum (Invitrogen; Thermo Fisher Scientific, Inc.), 2 mM 1-glutamine (Invitrogen; Thermo Fisher Scientific, Inc.), $100 \mathrm{U} / \mathrm{ml}$ penicillin (Invitrogen; Thermo Fisher Scientific, Inc.) and $100 \mu \mathrm{g} / \mathrm{ml}$ streptomycin (Invitrogen; Thermo Fisher Scientific, Inc.) at $37^{\circ} \mathrm{C}$ in humidified air containing 5\% $\mathrm{CO}_{2}$. An S6K2 short interfering (si)RNA oligonucleotide, 5'-GGAAGAAAACCAUGGAUA AUU-3' and its complementary RNA were synthesized by Genepharm, Inc. (Sunnyvale, CA, USA). Non-silencing control siRNA was synthesized by Genepharm using a scrambled sequence as the negative control. For transfection, siRNA or a plasmid were transfected into HCT116 cells using Lipofectamine ${ }^{\circledR} 2000$ (Invitrogen; Thermo Fisher Scientific, Inc.), according to manufacturer's instructions. The sequence of an antisense oligonucleotide (ASO) against exon 10 for the
PKM gene was 5'-TGAGGAACTCCGCCG-3'. DASA-58 was purchased from Cayman Chemical Company (Ann Arbor, MI, USA). Dimethyl sulfoxide (DMSO) was used as the vehicle control for DASA-58. Cells were treated with DMSO or $50 \mu \mathrm{M}$ DASA-58 for $3 \mathrm{~h}$ at $37^{\circ} \mathrm{C}$ in humidified air containing $5 \% \mathrm{CO}_{2}$ prior to analysis. The antibody of serine 6 phosphorylation of hnRNPA1 was generated as described previously (20).

Patients and tissue specimens. The present retrospective study was conducted with the approval of the Ethics Committee of Zhongshan Hospital (Shanghai, China). All colorectal cancer tissues and adjacent non-cancerous tissues were obtained from 75 colorectal cancer patients between January 2002 and December 2008 in Zhongshan Hospital. Formalin-fixed, paraffin-embedded blocks of human tissues were re-cut to a thickness of $5 \mu \mathrm{m}$ and were subjected to immunostaining. There were 43 male and 32 female patients, with ages ranging between 26 and 83 years (median, 65 years). The median follow-up data was 56 months (range, 0-97 months). For the analyses of overall survival, survival time was defined as the period between the date of diagnosis and the date of mortality or the date of the last follow-up.

Immunohistochemical staining. Immunohistochemical staining on the paraffin-embedded sections was performed using an LSAB kit (Dako; Agilent Technologies, Inc., Santa Clara, CA, USA), as described previously (21). Briefly, tissue sections were de-waxed, hydrated and washed. After microwave antigen retrieval, the slides were treated with $3 \% \mathrm{H}_{2} \mathrm{O}_{2}$ for $10 \mathrm{~min}$ at room temperature to block endogenous peroxidase activity and then incubated overnight with the primary antibody of rabbit anti-serine 6 phosphorylation of hnRNPA1 (dilution, 1:200; antibody epitope, $\operatorname{MSKSES}(\mathrm{p})$ PKEPEQLRKL) at $4^{\circ} \mathrm{C}$. The sections were then incubated with the horseradish peroxidase-conjugated secondary antibody (dilution, 1:1,000; cat. no. ab6721; Abcam, Cambridge, $\mathrm{UK}$ ) for $1 \mathrm{~h}$ at room temperature. Signals were visualized using diaminobenzidine as the chromogen and counter-stained with hematoxylin ( $3 \mathrm{~min}$ at room temperature) and eosin (1 min at room temperature) Scoring was performed with the $\mathrm{H}$-score on the basis of the proportion of tumor cells staining at various intensities as follows: $0 x(\%$ tumor cells with no staining $)+1 \times(\%$ tumor cells with faint expression $)+2 x(\%$ tumor cells with moderate expression $)+3 x(\%$ tumor cells with strong expression). H-scores for specimens with multiple cores were averaged. High p-hnRNPA1-S6 level is defined as H-score equal or greater than the average score. Low p-hnRNPA1-S6 level staining is defined as $\mathrm{H}$-score less than the average score.

Reverse transcription-quantitative polymerase chain reaction $(R T-q P C R)$. Total RNA was isolated from HCT116 cells using the TRIzol reagent (Invitrogen; Thermo Fisher Scientific, Inc.), according to the manufacturer's protocol. Briefly, cells were lysed in a culture dish by adding TRIzol reagent. The RNA was precipitated from the aqueous phase by mixing with $0.2 \mathrm{ml}$ isopropyl alcohol per $1 \mathrm{ml}$ TRIzol reagent. RNA was precipitated by centrifugation at $12,000 \mathrm{x}$ g for $15 \mathrm{~min}$ at $4^{\circ} \mathrm{C}$. The RNA pellet was washed once with $75 \%$ ethanol. Subsequently, RNA was dissolved in RNase-free water. RNA concentration was measured using a Nanodrop spectrophotometer (Thermo 
Fisher Scientific, Inc.). CDNA was synthesized using the First-Strand cDNA Synthesis kit (GE Healthcare, Chicago, IL, USA), according to the manufacturer's protocol. The sequences of the primers used for the real-time PCR analysis were as follows. S6K2 sense, CGGGCTGAGAGGAACATTCTA and antisense, CTGGAAAGCATAGGCCAGTTCT; PKM1 sense, CTGGAGAAACAGCCAAAGG and antisense, GCC AGACTCCGTCAGAACTA; PKM2 sense, GGGTTCGGA GGTTTGATG and antisense, ACGGCGGTGGCTTCTGT; $\beta$-actin sense, ATGGATGACGATATCGCTGCGC and antisense, GCAGCACAGGGTGCTCCTCA. Real-time PCR was performed with the Sequence Detection system 7900HT (Applied Biosystems; Thermo Fisher Scientific, Inc.). Thermal cycling conditions were designed as follows: Initial denaturation at $95^{\circ} \mathrm{C}$ for $10 \mathrm{~min}$, followed by 40 cycles of $95^{\circ} \mathrm{C}$ for $15 \mathrm{sec}$ and $60^{\circ} \mathrm{C}$ for $1 \mathrm{~min}$. Threshold cycle $(\Delta \mathrm{CT})$ values were calculated by subtracting the $\mathrm{CT}$ value from the corresponding $\beta$-actin CT (internal control) value. The comparative $\mathrm{Cq}$ $(\Delta \Delta \mathrm{Cq})$ method was used to determine the expression level of mRNA (22).

Apoptosis analysis. Cell apoptosis was analyzed using the Annexin V-fluorescein isothiocyanate Apoptosis Detection kit (Sigma-Aldrich; Merck KGaA, Darmstadt, Germany). Cells were harvested by treating with trypsin. As described previously (23), cells were washed with ice-cold PBS, resuspended in the Annexin V binding buffer and, following incubation, cells were analyzed using a FACStarPlus flow cytometer (BD Biosciences, Franklin lakes, NJ, USA). Data were collected and analyzed using CellQuest software (version 5.1; BD Biosciences).

Western blot analysis. As described in detail previously (24), cells were washed twice in PBS, suspended in lysis buffer (50 mM Tris at $\mathrm{pH} 8.0,150 \mathrm{mM} \mathrm{NaCl}, 0.1 \%$ SDS, $0.5 \%$ sodium deoxycholate, $1 \%$ Nonidet P-40, phenylmethylsulfonyl fluoride at $100 \mu \mathrm{g} / \mathrm{ml}$, aprotinin at $2 \mu \mathrm{g} / \mathrm{ml}$, pepstatin at $1 \mu \mathrm{g} / \mathrm{ml}$ and leupetin at $10 \mu \mathrm{g} / \mathrm{ml}$ ) and placed on ice for $30 \mathrm{~min}$. Following centrifugation at $15,000 \mathrm{x}$ g for $20 \mathrm{~min}$ at $4^{\circ} \mathrm{C}$, the suspension was collected. Protein concentration was estimated using the BCA protein assay kit (Thermo Fisher Scientific, Inc.). Proteins $(20 \mu \mathrm{g})$ were resolved using 12\% SDS-PAGE and transferred to polyvinylidne difluoride membranes. The membranes were blocked in $5 \%$ non-fat milk in TBST $(0.1 \%$ Tween in TBS) for $1 \mathrm{~h}$ at room temperature. Proteins were probed with mouse anti-flag antibody (1:1,000 dilution, cat. no. F3165; Sigma-Aldrich; Merck KGaA), rabbit anti-phoshposerine (1:1,000 dilution, cat. no. ab9332; Abcam) for $3 \mathrm{~h}$ at room temperature and followed by incubation with a horseradish peroxidase-conjugated secondary antibody (1:10,000 dilution; cat. no. ab9332; Abcam) for $1 \mathrm{~h}$ at room temperature. The bands were visualized using enhanced chemiluminescence reagents (Thermo Fisher Scientific, Inc.).

Chromatin immunoprecipitation assay (ChIP). ChIP was performed using the EZ-ChIP Assay kit (EMD Millipore, Billerica, MA, USA), according to manufacturer's instructions. HCT116 cells $\left(2 \times 10^{6}\right)$ were transfected with siRNA against S6K2 or non-silencing control for $48 \mathrm{~h}$ at $37^{\circ} \mathrm{C}$. The cells were cross-linked with $1 \%$ formaldehyde for $10 \mathrm{~min}$ at room temperature and the reaction was quenched using $0.125 \mathrm{M}$ glycine. Cells were washed twice using ice cold PBS containing protease inhibitors. Cells were pelleted for $4 \mathrm{~min}$ at $500 \mathrm{x} \mathrm{g}$ and $4^{\circ} \mathrm{C}$. Cell pellets were resuspended in SDS Lysis Buffer (cat. no. 20-163; EMD Millipore). The lysate was sonicated to obtain DNA fragments between 200 and 1,000 basepairs in length and validated using electrophoresis on $1 \%$ agarose gels. The sheared DNA was centrifuged at $12,000 \mathrm{x} \mathrm{g}$ at $4^{\circ} \mathrm{C}$ for $10 \mathrm{~min}$. Soluble chromatin was immunoprecipitated overnight with mouse anti- hnRNP A1 antibody (5 $\mu \mathrm{g}$; cat. no. sc-32301; Santa Cruz Biotechnology, CA, USA) overnight at $4^{\circ} \mathrm{C}$. Immunocomplexes were washed once in $1 \mathrm{ml}$ Low Salt Immune Complex Wash Buffer (cat. no. 20-154; EMD Millipore), once in $1 \mathrm{ml}$ High Salt Immune Complex Wash Buffer (cat. no. 20-155; EMD Millipore), once in $1 \mathrm{ml}$ LiCl Immune Complex Wash Buffer (cat. no. 20-156; EMD Millipore), twice in $1 \mathrm{ml}$ TE Buffer (cat. no. 20-157; EMD Millipore). Following reversal and recovery of the immunoprecipitated chromatin DNA, the final DNA pellets were dissolved in $50 \mu \mathrm{l} \mathrm{H}_{2} \mathrm{O}$. The purified DNA samples were used as templates for semi-quantitative and qPCR to determine the relative enrichment.

qPCR was performed using SYBR Green Real-Time PCR Master mix (Thermo Fisher Scientific, Inc.). The comparative $\mathrm{Cq}(\Delta \Delta \mathrm{Cq})$ method was used to determine the expression level of mRNA (22). Thermal cycling conditions were designed as follows: Initial denaturation at $95^{\circ} \mathrm{C}$ for $10 \mathrm{~min}$, followed by 40 cycles of $95^{\circ} \mathrm{C}$ for $15 \mathrm{sec}$ and $60^{\circ} \mathrm{C}$ for $1 \mathrm{~min}$. The sequences of the primers were: EI9 sense, TCCTAGAGCAGGTGGAGCAA and anti-sense, CTGACGGAGTCTGGCAGGTA; $\beta$-actin sense, ATGGATGACGATATCGCTGCGC and antisense, GCAGCACAGGGTGCTCCTCA. $\beta$-actin was used as a reference gene.

Immunoprecipitation. HCT116 cells were transfected with wild-type Flag-hnRNPA1, S6A or S4A mutant of Flag-hnRNPA1 for $48 \mathrm{~h}$ at $37^{\circ} \mathrm{C}$ in humidified air containing $5 \% \mathrm{CO}_{2}$. Cells were collected and protein were extracted as described previously. Subsequently, cell lysates were incubated with anti-FLAG-M2 beads (Sigma-Aldrich; Merck $\mathrm{KGaA}$ ) for $3 \mathrm{~h}$ at $4^{\circ} \mathrm{C}$ with constant rotation. The beads were washed 5 times with buffer $(50 \mathrm{mM}$ Tris $\mathrm{HCl}, 150 \mathrm{mM} \mathrm{NaCl}$, $1 \mathrm{mM}$ EDTA and 1\% Triton X-100). The immunocomplexes were then eluted with $3 \mathrm{X}$ Flag peptide (Sigma-Aldrich; Merck $\mathrm{KGaA}$ ) in $0.5 \mathrm{M}$ Tris $\mathrm{HCl}(\mathrm{pH} 7.5)$ with $1 \mathrm{M} \mathrm{NaCl}$ for $30 \mathrm{~min}$ at $4^{\circ} \mathrm{C}$. The beads were centrifuged for $30 \mathrm{sec}$ at $8,000 \mathrm{x} \mathrm{g}$ at $4^{\circ} \mathrm{C}$ to collect supernatants for western blot analysis. Western blot analysis was performed as described previously.

Glucose uptake assay. Glucose uptake in cells was quantified using a Glucose Uptake Assay kit (BioVision, Inc., Milpitas, CA, USA) Briefly, cells were cultured in glucose-free DMEM for $16 \mathrm{~h}$ at $37^{\circ} \mathrm{C}$ and then incubated with high-glucose DMEM for an additional $24 \mathrm{~h}$ at $37^{\circ} \mathrm{C}$. The culture medium was removed, and the intracellular glucose levels were evaluated according to the kit manufacturer's instructions.

Statistical analysis. All experiments were performed in triplicate and the results are presented as the mean \pm standard deviation. The significance of differences between 
A

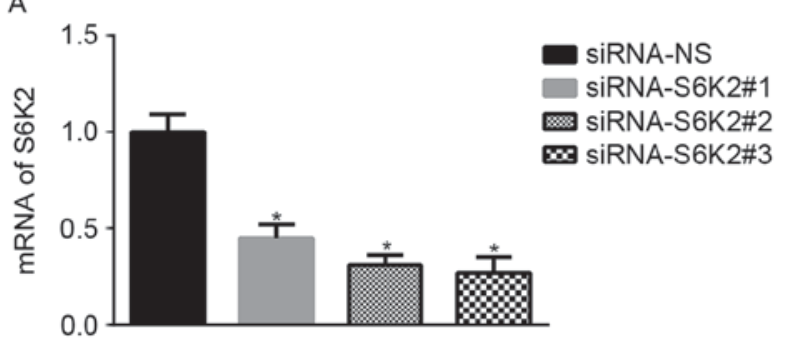

$\mathrm{C}$

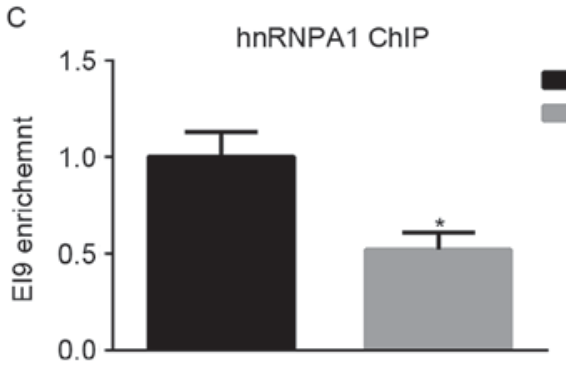

E

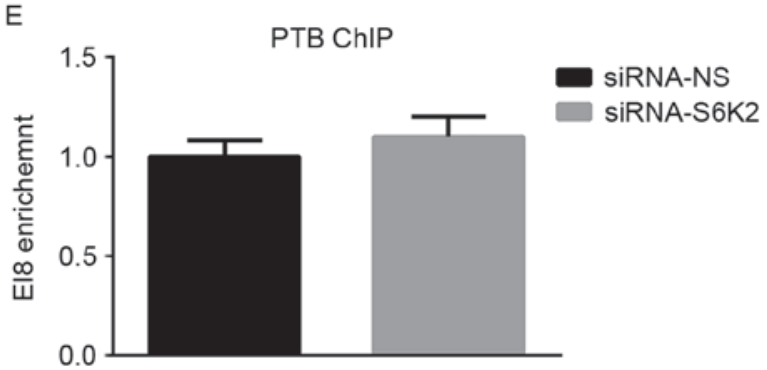

B

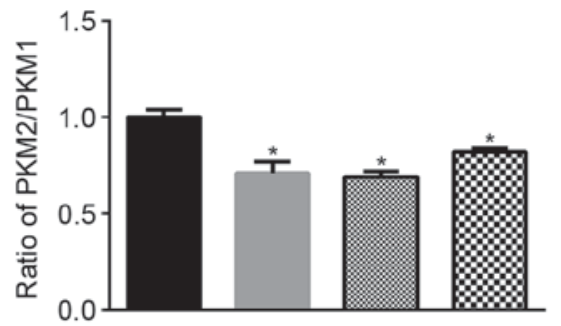

SiRNA-NS

SiRNA-S6K2

D

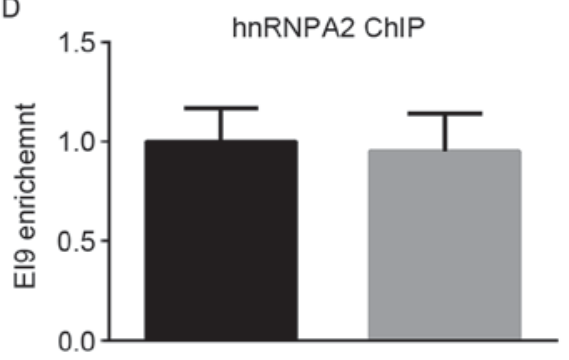

SiRNA-NS

SiRNA-S6K2\#1

SiRNA-S6K2\#2

siRNA-S6K2\#3

SiRNA-NS

SiRNA-S6K2

Figure 1. S6K2 regulates alternative splicing of the PKM gene. (A) HCT116 cells were transfected with siRNAs against S6K2 or non-silencing control. The mRNA levels of S6K2 were analyzed using qPCR. (B) HCT116 cells were transfected with siRNAs against S6K2 or NS control. The mRNA levels of PKM1 or PKM2 were analyzed using qPCR. The ratio of PKM2/PKM1 was calculated according to their mRNA levels. HCT116 cells were transfected with siRNA against S6K2 or non-silencing control. (C) hnRNPA1, (D) hnRNPA2 and (E) PTB antibodies were used for chromatin immunoprecipitation analysis. The primers recognizing EI9 or EI8 splicing site were used for analyzing enrichment of indicated protein. Data are presented as the mean \pm standard deviation. ${ }^{\text {"P }}<0.05$ vs. siRNA non-silencing control. S6K2, S6 kinase 2; PKM, pyruvate kinase; PKM1, pyruvate kinase M1 isoform; PKM2, pyruvate kinase M2 isoform; siRNA, short interfering RNA; qPCR, quantitative polymerase chain reaction; hnRNPA1, heterogeneous nuclear ribonucleoprotein A1; hnRNPA2, heterogeneous nuclear ribonucleoprotein A2; -NS, non-silencing; PTB, RNA binding motif containing.

experimental groups was analyzed using the unpaired Student's t-test or one-way analysis of variance followed by Student Newman Keuls post hoc test. $\mathrm{P}<0.05$ was considered to indicate a statistically significant difference.

\section{Results}

S6K2 regulates alternative splicing of PKM gene in a hnRNPAl-dependent manner. To obtain an insight into the function of S6K2 in alternative splicing of the PKM gene, three siRNAs were used to knockdown S6K2 expression in the CRC cell line HCT116 (Fig. 1A). The mRNA ratio of PKM2/PKM1 was downregulated by S6K2 knockdown (Fig. 1B). Binding of hnRNPA1, hnRNPA2 and PTB to the flanking regions of exon 9 result in the exclusion of exon 9 and subsequent expression of the PKM2 isoform (7). Therefore, it was investigated whether S6K2 may affect the binding of hnRNPA1, hnRNPA2 and PTB to splicing sites of the PKM gene. ChIP analysis was applied to determine the abundance of hnRNPA1, hnRNPA2 and PTB on the PKM gene in HCT116 cells. Compared with non-silencing control, S6K2 knockdown decreased hnRNPA1 binding to the downstream exon 9 splice site (EI9; Fig. 1C), whereas binding of hnRNPA2 to the corresponding region was unaffected (Fig. 1D). PTB binding to the upstream exon 9 splicing site (EI8) was unchanged following S6K2 knockdown (Fig. 1E). Therefore, the results of the present study indicated that S6K2 was involved in alternative splicing of PKM gene by modulating hnRNPA1 binding to the splicing site.

Serine 6 phosphorylation of hnRNPA1 is required for S6K2 regulating the levels of PKM isoforms. hnRNPA1 has been identified as a substrate of S6K2 kinase (19). Consistent with a previous study (19), mutation of either $\mathrm{Ser}^{4}$ or $\mathrm{Ser}^{6}$ residue from serine to alanine decreased serine phosphorylation of hnRNPA1 (Fig. 2A). Subsequently, the present study aimed to determine whether this phosphorylation event was involved in alternative splicing of the PKM gene. hnRNPA1 may induce the expression of PKM2 and inhibit the expression of PKM1 (5). The results of the present study demonstrated that the PKM2/PKM1 mRNA ratio was increased by hnRNPA1 in HCT116 cells compared with the vector control (Fig. 2B). In contrast with the wild-type, hnRNPA1-S6A mutant was unable to increase the mRNA ratio of PKM2/PKM1 (Fig. 2B). In addition, the mRNA ratio of PKM2/PKM1 did not significantly differ between the wild-type and hnRNPA1-S4A mutant (Fig. 2B). Furthermore, mutation at residue $\mathrm{Ser}^{6}$ inhibited the decrease of the PKM2/PKM1 mRNA 
A

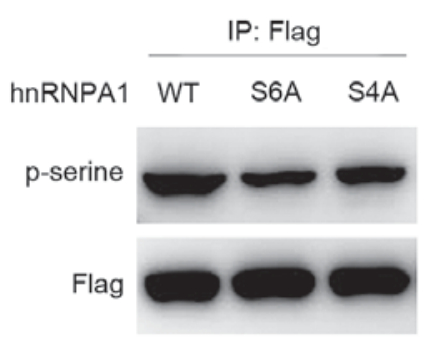

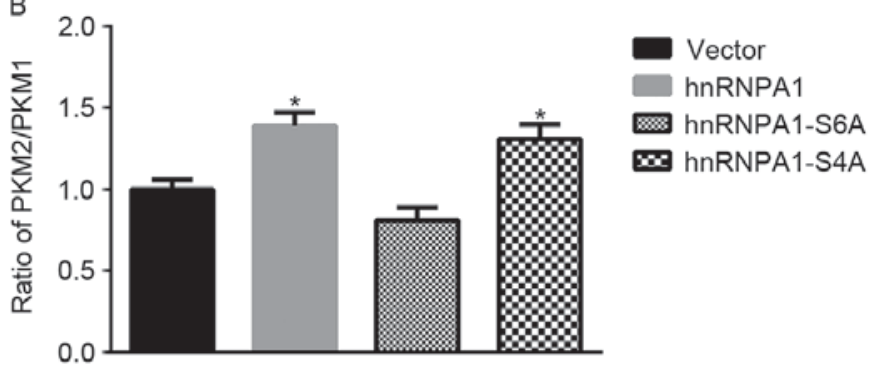

D

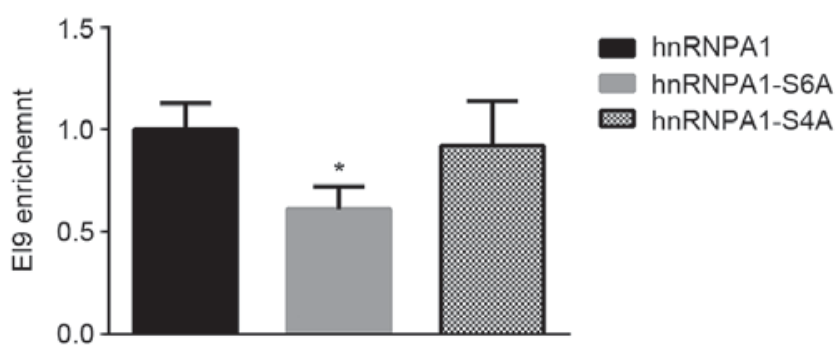

hnRNPA1

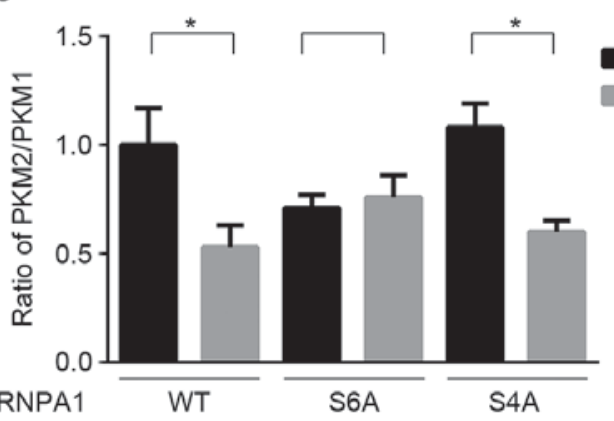

siRNA-NS siRNA-S6K2

Figure 2. Serine 6 phosphorylation of hnRNPA1 by S6K2 regulates PKM expression. (A) HCT116 cells were transfected with wild-type Flag-hnRNPA1, S6A or S4A mutant of Flag-hnRNPA1. Flag antibodies were used for immunoprecipitation. The phosphor-serine antibody was applied for determining the serine phosphorylation in hnRNPA1. (B) HCT116 cells were transfected with empty vector, wild-type Flag-hnRNPA1, and S6A or S4A mutant of Flag-hnRNPA1. The ratio of PKM2/PKM1 was calculated according to their mRNA levels, as analyzed using qPCR. *P<0.05 vs. vector control. (C) HCT116 cells were co-transfected with siRNA against S6K2 (NS siRNA as the control) and empty vector, wild-type Flag-hnRNPA1, S6A or S4A mutant of Flag-hnRNPA1. The ratio of PKM2/PKM1 was calculated according to their mRNA levels, as analyzed using qPCR. * $\mathrm{P}<0.05$ vs. siRNA non-silencing control. (D) HCT116 cells were transfected with wild-type Flag-hnRNPA1 and S6A or S4A mutant of Flag-hnRNPA1. Flag antibodies were used for chromatin immunoprecipitation analysis. The primers recognizing EI9 splicing site were used for analyzing enrichment of indicated protein. ${ }^{*} \mathrm{P}<0.05$ vs. hnRNPA1 group. Data are presented as the mean \pm standard deviation. hnRNPA1, heterogeneous nuclear ribonucleoprotein A1; PKM, pyruvate kinase; S6A, serine 6 A; S4A, serine 4 A; PKM2, pyruvate kinase M2 isoform; PKM1, pyruvate kinase M1 isoform; qPCR, quantitative polymerase chain reaction; siRNA, short interfering RNA; WT, wild-type; -NS, non-silencing.

ratio induced by S6K2 knockdown (Fig. 2C). Only Ser ${ }^{6}$ phosphorylation of hnRNPA1 is required for alternative splicing of the PKM gene, in spite of $\mathrm{Ser}^{4}$ and $\mathrm{Ser}^{6}$ being phosphorylating sites of S6K2 (16). Subsequently, the present study aimed at identifying whether $\mathrm{Ser}^{6}$ phosphorylation of hnRNPA1 by S6K2 affected the mRNA ratio of PKM2/PKM1. ChIP analysis indicated that the abundance of hRNPA1 on the flanking regions of exon 9 was decreased in HCT116 cells transfected with hnRNPA1-S6A mutant, compared with that transfected with either wild-type or hnRNPA1-S4A mutant (Fig. 2D). Therefore, the results of the present study indicated that S6K2 selectively phosphorylates $\operatorname{Ser}^{6}$ residue of hnRNPA1 to regulate alternative splicing of PKM gene.

S6K2 promotes glycolysis of CRC cells via hnRNPA1-mediated alterative splicing of PKM gene. On the basis of the fundamental function of PK in glycolysis, the present study aimed at determining whether S6K2 may regulate glycolysis of CRC cell lines. S6K2 knockdown decreased glycolysis, as indicated by glucose consumption (Fig. 3A) or lactate production (Fig. 3B). S6K2-mediated Ser ${ }^{6}$ phosphorylation was inhibited by expression of the hnRNPA1-S6A mutant, but S6K2 did not exhibit a regulatory effect on glycolysis of HCT116 cells (Fig. 3C and D). To identify the function of hnRNPA1-mediated splicing in glucose metabolism, an ASO against exon 10 for PKM gene was used. Consistent with a previous study (25), this specific ASO against exon 10 decreased the mRNA ratio of PKM2/PKM1 (Fig. 3E). Furthermore, the decrease of
PKM2/PKM1 induced by S6K2 knockdown was inhibited in the presence of the ASO against exon 10 (Fig. 3E). In the presence of ASO against exon10, the S6K2-mediated glycolysis was inhibited (Fig. 3F and G). The results of the present study suggested that S6K2 modulated glycolysis of CRC cells by phosphorylating the $\operatorname{Ser}^{6}$ residue of hnRNPA1.

S6K2 promotes proliferation of CRC cells through activating glycolysis. Subsequently, the present study investigated whether the metabolic advantages induced by S6K2 regulated the proliferation of CRC cells. S6K2 knockdown significantly decreased the growth of HCT116 cells (Fig. 4A). However, the proportion of apoptotic cells increased following silencing of S6K2 in HCT116 cells (Fig. 4B). HCT116 cells transfected with hnRNPA1 mutant devoid of S6K2-mediated Ser ${ }^{6}$ phosphorylation exhibited a decreased fold-change in the cell count, compared with those transfected with wild-type hnRNPA1 (Fig. 4C). To understand the function of glucose metabolism in S6K2-mediated proliferation of cancer cells, a PKM2 activator, DASA-58, was used to modulate glycolysis. DASA-58 inhibits proliferation and glycolysis of cancer cells by activating PKM2 enzyme activity (26). Glucose consumption (Fig. 4D) and lactate production (Fig. 4E) of HCT116 cells were inhibited by DASA-58. In the presence of DASA-58, the effect of S6K2 knockdown on cell growth was inhibited (Fig. 4F). Therefore, the results of the present study demonstrated that serine 6 phosphorylation of hnRNPA1 regulated cancer cell growth, which is associated with PKM2-mediated glycolysis. 

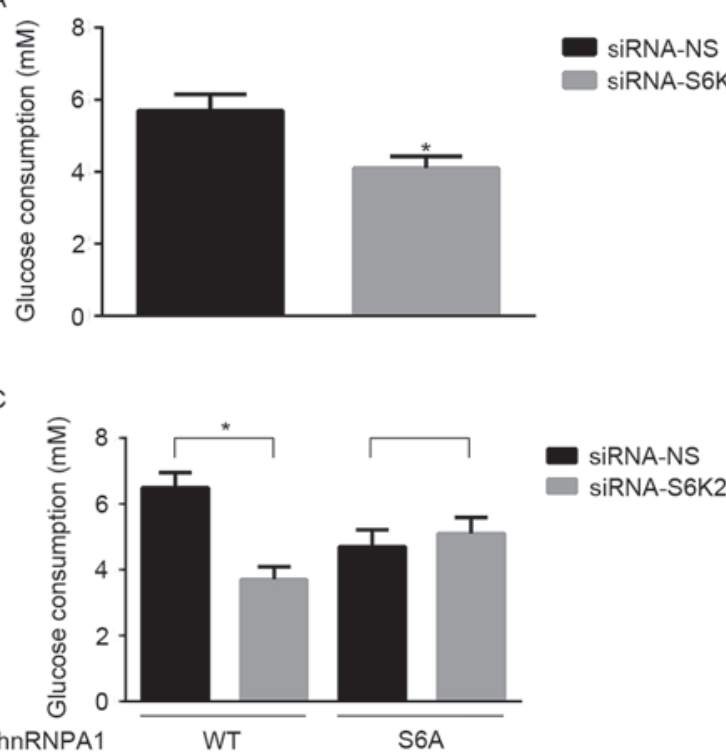

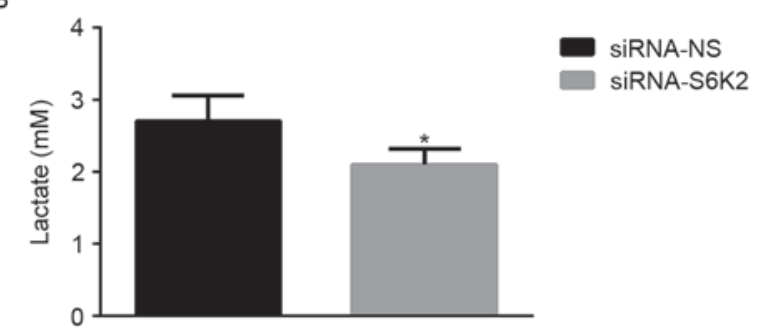

D

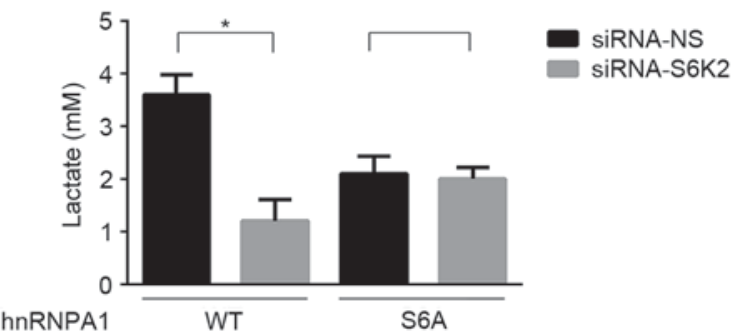

$E$

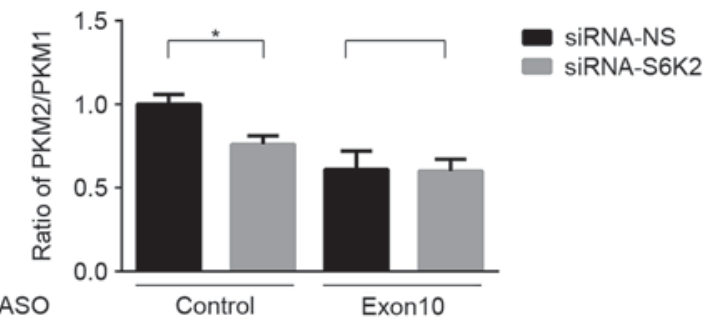

F

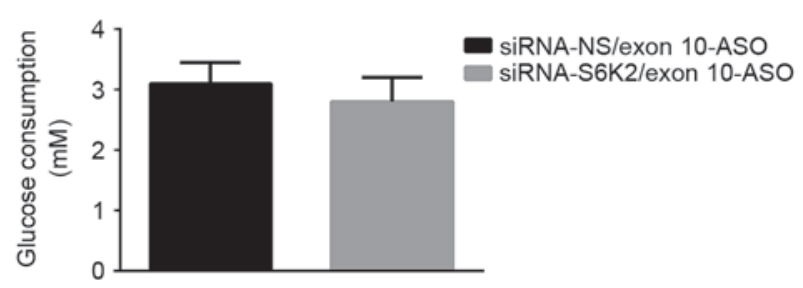

G

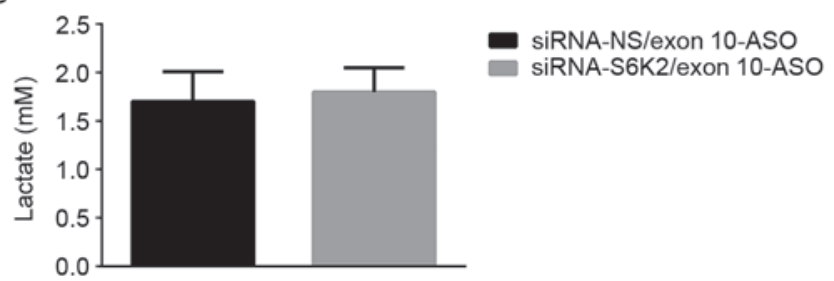

Figure 3. S6K2 promotes glycolysis of CRC cells. HCT116 cells were transfected with siRNA against S6K2 or NS control. (A) Glucose consumption and (B) lactate production were analyzed in these cells. HCT116 cells were co-transfected with siRNA against S6K2 (NS siRNA as the control) and wild-type Flag-hnRNPA1, and S6A or S4A mutant of Flag-hnRNPA1. (C) Glucose consumption and (D) lactate production were analyzed in these cells. (E) HCT116 cells were co-transfected with siRNA against S6K2 (NS siRNA as the control) and EI9-ASO against EI9 splicing site or control ASO. The ratio of PKM2/PKM1 was calculated according to their mRNA level as analyzed by qPCR. HCT116 cells were co-transfected with siRNA against S6K2 (NS siRNA as the control) and EI9-ASO against EI9 splicing site or control ASO. (F) Glucose consumption and (G) lactate production were analyzed in these cells. Data are presented as the mean \pm standard deviation. "P<0.05 vs. siRNA non-silencing control. S6K2, S6 kinase 2; CRC, colorectal cancer; siRNA, short interfering RNA, -NS, non-silencing; hnRNPA1, heterogeneous nuclear ribonucleoprotein A1; S6A, serine 6 A; S4A, serine 4 A; ASO, antisense oligonucleotide; qPCR, quantitative polymerase chain reaction.

hnRNPA1 phosphorylation by $S 6 K 2$ predicts poor prognosis of $C R C$. To analyze the clinical significance of serine 6 phosphorylation of hnRNPA1, a specific antibody against this modification was generated (Fig. 5A). Subsequently, IHC analysis was performed in 75 colorectal cancer tissues. Representative staining images of phosphorylation of hnRNPA1-S6 are presented in Fig. 5B. According to semi-quantitative analysis of the level of serine 6 phosphorylation of hnRNPA1, patients were divided into two groups: High $(n=30)$ and low $p-h n R N P A 1-S 6$ level $(n=45)$ groups. The median survival duration was 44.633 months [ $95 \%$ confidence interval (CI), 32.328-56.938] and 61.822 months (95\% CI, 50.984-72.660) for the high and low p-hnRNPA1-S6 groups, respectively. The Kaplan-Meier estimator survival analysis indicated that patients belonging to the high p-hnRNPA1-S6 group exhibited a significantly decreased overall survival time, compared with those classified as the low p-hnRNPA1-S6 group (Fig. 5C). Therefore, the results of the present study suggested that serine 6 phosphorylation of hnRNPA1 may be a useful prognostic marker for patients with CRC.

\section{Discussion}

CRC is one of the most common types of cancer with high morbidity and mortality rates worldwide (27). In addition to genetic and epigenetic alterations in oncogenes and 
A

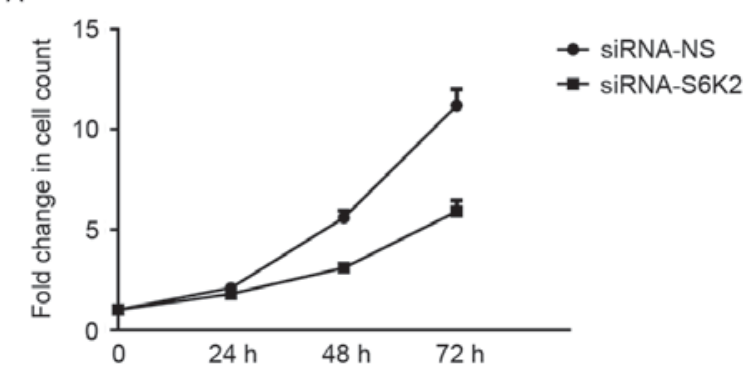

C

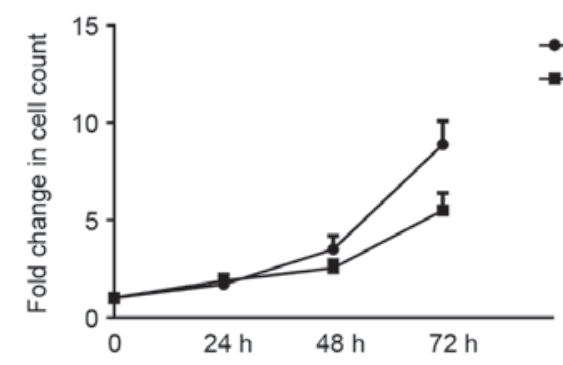

E

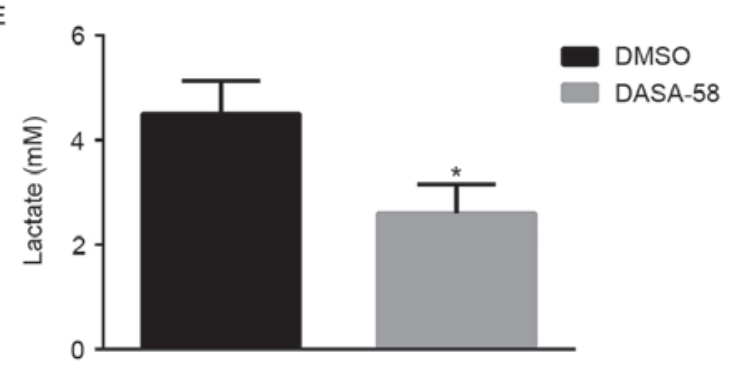

B

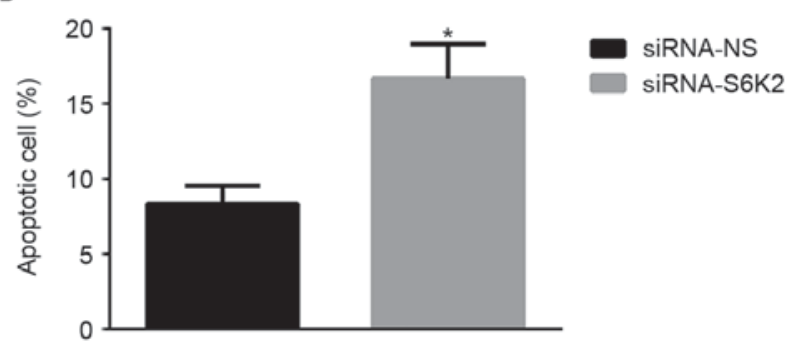

D

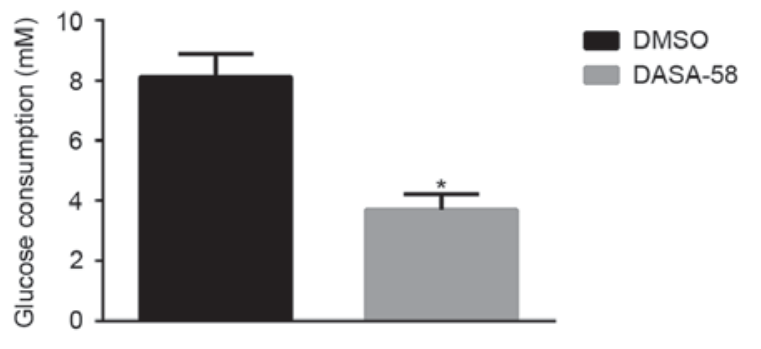

F

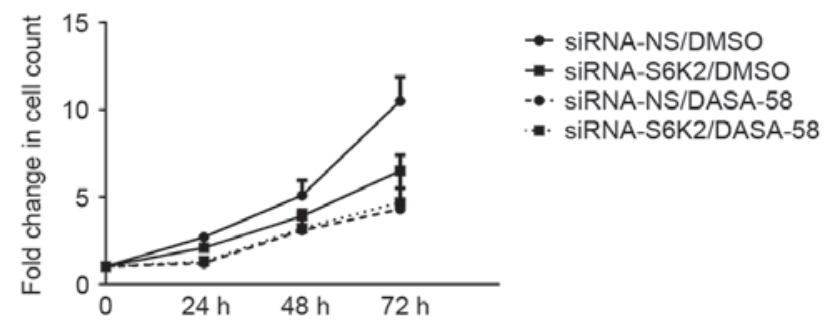

Figure 4. S6K2 promotes proliferation of CRC cells. (A) HCT116 cells were transfected with siRNA against S6K2 or NS control. Cell numbers were counted every $24 \mathrm{~h}$ for 3 days. (B) Fluorescence-activated cell sorting analysis revealed the proportion of apoptotic cells in HCT116 cells transfected with siRNA against S6K2 or NS control. "P<0.05 vs. siRNA non-silencing control. (C) HCT116 cells were transfected with wild-type Flag-hnRNPA1 and S6A mutant of Flag-hnRNPA1. Cell numbers were counted every $24 \mathrm{~h}$ for 3 days. HCT116 cells were treated with DASA-58 or DMSO. (D) Glucose consumption and (E) lactate production were analyzed in these cells. "P<0.05 vs. DMSO control. (F) HCT116 cells were transfected with siRNA against S6K2 or NS control. Subsequently, cells were treated with DASA-58 or DMSO. Cell numbers were counted every $24 \mathrm{~h}$ for 3 days. Data are presented as the mean \pm standard deviation. S6K2, S6 kinase 2; CRC, colorectal cancer; siRNA, short interfering RNA; -NS, non-silencing; hnRNPA1, heterogeneous nuclear ribonucleoprotein A1; DMSO, dimethyl sulfoxide.

tumor suppressors, metabolic disturbance has an effect on the initiation and progression of CRC (28). The reprogramming of glucose metabolism is observed in a number of types of cancer, including CRC (4). The Warburg effect, characterized as increased glucose consumption and lactate production, is the metabolic phenotype observed in CRC (29). An increase in glucose metabolism by glycolysis, rather than by oxidative phosphorylation, promotes tumor survival and chemoresistance in CRC (30). For this reason, understanding the underlying molecular mechanisms and potential targets involved in glucose metabolic reprogramming may enable the identification of novel therapeutic strategies for CRC. The results of the present study demonstrated that the phosphorylation of $\mathrm{Ser}^{6}$ in hnRNPA1 by S6K2 is required for glycolysis and the proliferation of CRC cells. In addition, the accumulation of $\mathrm{Ser}^{6}$ phosphorylation was associated with poor survival of patients with CRC.

Dysregulated glucose metabolism in CRC is regulated by a group of enzymes or proteins including glucose transporter 1, hexokinase 2, PKM2 and lactate dehydrogenase A. There is accumulating evidence demonstrating that PKM2 functions in glucose metabolic reprogramming in cancer cells $(31,32)$.
PKM2 levels are increased in CRC and associated with the tumor stage and lymph metastasis (33). Expressing PKM2 isoform, rather than the PKM1 isoform, is a typical characteristic observed during cancer development (34). In glioblastoma, the splicing factors (PTB, hnRNPA1 and hnRNPA2) and PKM2 are highly expressed (7). Therefore, inhibition of PKM2 expression by modulating alternative splicing of the PKM gene may be a useful strategy to treat cancer. The results of the present study revealed that S6K2 is an upstream regulator of the alternative splicing of the PKM gene, by phosphorylating hnRNPA1. On the basis of the results of the present study, the pan-S6 K inhibitor or specific S6K2 inhibitor may be exploited for CRC treatment.

hnRNPA1, as a RNA binding protein, participates in various steps of maturation of nascent transcripts (35). hnRNPA1 participates in alternative splicing of multiple genes. The binding of hnRNPA1 to a transcript of BRCA1 DNA repair associated results in exon 18 skipping (36) and hnRNPA1 has been identified to repress exon 3 inclusion during transcription of human immunodeficiency virus type 1 (1). In regard to alternative splicing of the PKM gene, hnRNPA1 binds to the flanking of exon 9, which causes exon 10 inclusion and exon 9 
A

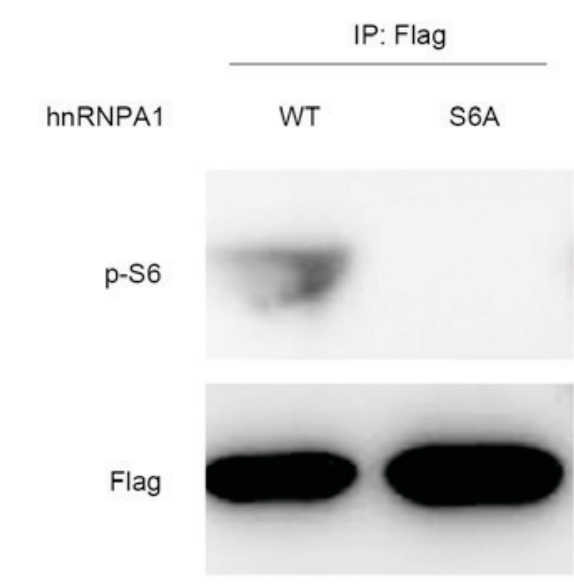

B
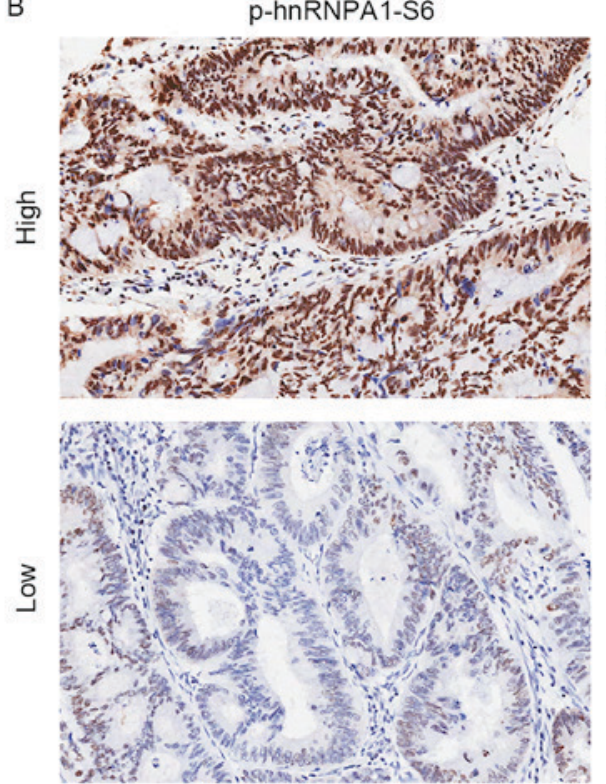
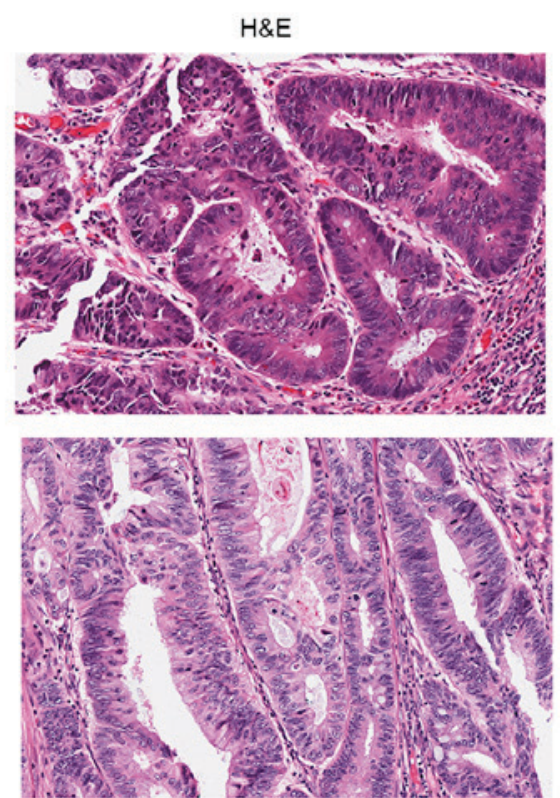

C

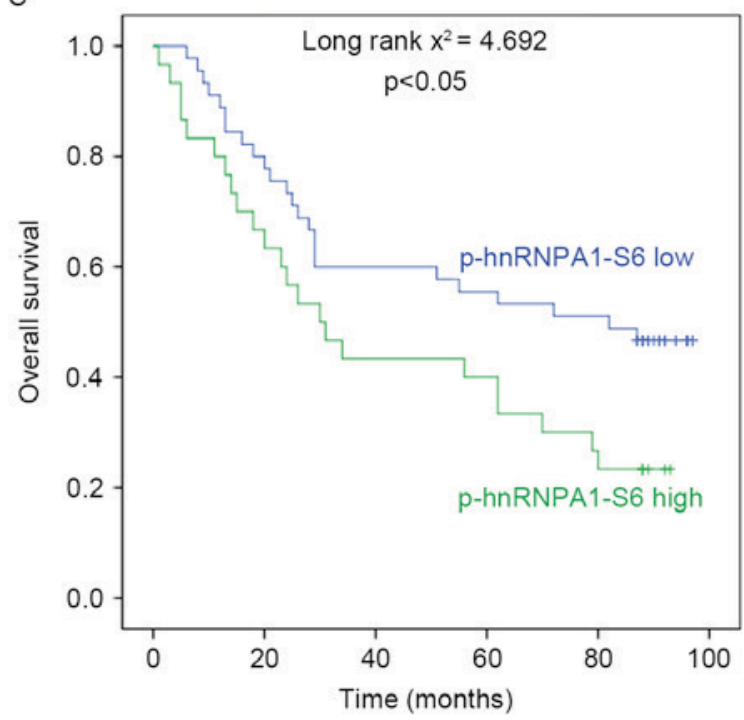

Figure 5. hnRNPA1 phosphorylation at serine 6 residue is associated with prognosis of CRC. (A) HCT116 cells were transfected with wild-type Flag-hnRNPA1 and S6A mutant of Flag-hnRNPA1. Flag antibodies were used for immunoprecipitation. The specific hnRNPA1-Ser ${ }^{6}$ phosphorylation antibody was applied to determine serine phosphorylation in hnRNPA1. (B) Representative immunohistochemistry images (magnification, x10) of hnRNPA1 phosphorylation at serine 6 residue in CRC tissues, by the specific hnRNPA1-Ser ${ }^{6}$ phosphorylation antibody. (C) Kaplan-Meier estimator overall survival, according to the level of hnRNPA1 phosphorylation at serine 6 residue. hnRNPA1, heterogeneous nuclear ribonucleoprotein A1; CRC, colorectal cancer; H\&E, hematoxylin and eosin staining; p-S6, phosphorylated serine 6; S6A, serine 6A.

exclusion (7). Oncogenic transcription factor c-MYC promotes hnRNPA1 expression and subsequent alternative splicing of PKM gene (7). Ser6/4 in hnRNPA1 are phosphorylation sites of S6K2 kinase and the phosphorylation on these two sites is involved in the translation of BCL-xL and XIAP (19). Notably, the results of the present study indicated that only $\mathrm{Ser}^{6}$ phosphorylation is required for promoting hnRNPA1 to bind to the splicing site of the PKM gene. Ser ${ }^{6}$, rather than $\mathrm{Ser}^{4}$, phosphorylation may cause structural alterations to hnRNPA1, which facilitated the binding of $\operatorname{Ser}^{6}$ to the splicing site of the PKM gene. In addition, this suggested that the phosphorylation pattern of hnRNPA1 by S6K2 may be distinct in regard to the specific target gene of hnRNPA1.

The results of the present study provided an insight into how CRC cells may reprogramme glucose metabolism to maintain its malignant phenotype. It was demonstrated that the phosphorylation of hnRNPA1 by S6K2 is required for glycolysis and growth of CRC cells. Inhibiting S6K2-meidated modification of hnRNPA1 may be a future strategy for CRC treatment.

\section{Acknowledgements}

The present study was supported by the National Natural Science Foundation of China (grant nos. 81201532, 81372195 and 81572719), the Science and Technology Commission of Shanghai Municipality (grant no. 134119a5600), the Program for Professor of Special Appointment (Eastern Scholar) at Shanghai Institutions of Higher Learning (grant no. 1410000157) and the Shanghai Municipal Commission of Health and Family Planning (grant no. XYQ2013109). 


\section{References}

1. Tange TO, Damgaard CK, Guth S, Valcárcel J and Kjems J: The hnRNP A1 protein regulates HIV-1 tat splicing via a novel intron silencer element. EMBO J 20: 5748-5758, 2001.

2. Cunningham D, Humblet $Y$, Siena S, Khayat D, Bleiberg H, Santoro A, Bets D, Mueser M, Harstrick A, Verslype C, et al Cetuximab monotherapy and cetuximab plus irinotecan in irinotecan-refractory metastatic colorectal cancer. N Engl J Med 351: 337-345, 2004

3. Hanahan D and Weinberg RA: Hallmarks of cancer: The next generation. Cell 144: 646-674, 2011

4. Koppenol WH, Bounds PL and Dang CV: Otto Warburg's contributions to current concepts of cancer metabolism. Nat Rev Cancer 11: 325-337, 2011

5. Lunt SY and Vander Heiden MG: Aerobic glycolysis: Meeting the metabolic requirements of cell proliferation. Annu Rev Cell Dev Biol 27: 441-464, 2011.

6. Mazurek S, Boschek CB, Hugo F and Eigenbrodt E: Pyruvate kinase type M2 and its role in tumor growth and spreading. Semin Cancer Biol 15: 300-308, 2005.

7. David CJ, Chen M, Assanah M, Canoll P and Manley JL: HnRNP proteins controlled by c-Myc deregulate pyruvate kinase mRNA splicing in cancer. Nature 463: 364-368, 2010.

8. Yang W, Xia Y, Hawke D, Li X, Liang J, Xing D, Aldape K, Hunter T, Alfred Yung WK and Lu Z: PKM2 phosphorylates histone $\mathrm{h} 3$ and promotes gene transcription and tumorigenesis. Cell 150: 685-696, 2012.

9. Luo W, Hu H, Chang R, Zhong J, Knabel M, O'Meally R, Cole RN, Pandey A and Semenza GL: Pyruvate kinase M2 is a PHD3-stimulated coactivator for hypoxia-inducible factor 1. Cell 145: 732-744, 2011

10. Christofk HR, Vander Heiden MG, Harris MH, Ramanathan A Gerszten RE, Wei R, Fleming MD, Schreiber SL and Cantley LC: The M2 splice isoform of pyruvate kinase is important for cancer metabolism and tumour growth. Nature 452: 230-233, 2008.

11. Calabretta S, Bielli P, Passacantilli I, Pilozzi E, Fendrich V, Capurso G, Fave GD and Sette C: Modulation of PKM alternative splicing by PTBP1 promotes gemcitabine resistance in pancreatic cancer cells. Oncogene 35: 2031-2039, 2016.

12. Sun Y, Zhao X, Zhou Y and Hu Y: miR-124, miR-137 and miR-340 regulate colorectal cancer growth via inhibition of the Warburg effect. Oncol Rep 28: 1346-1352, 2012.

13. Pearce LR, Komander D and Alessi DR: The nuts and bolts of AGC protein kinases. Nat Rev Mol Cell Biol 11: 9-22, 2010.

14. Magnuson B, Ekim B and Fingar DC: Regulation and function of ribosomal protein S6 kinase (S6K) within mTOR signalling networks. Biochem J 441: 1-21, 2012.

15. Pardo OE and Seckl MJ: S6K2: The neglected s6 kinase family member. Front Oncol 3: 191, 2013.

16. Savinska LO, Lyzogubov VV, Usenko VS, Ovcharenko GV, Gorbenko ON, Rodnin MV, Vudmaska MI, Pogribniy PV Kyyamova RG, Panasyuk GG, et al: Immunohistochemical analysis of S6K1 and S6K2 expression in human breast tumors. Eksp Onkol 26: 24-30, 2004.

17. Lyzogubov VV, Lytvyn DI, Dudchenko TM, Lubchenko NV, Pogrybniy PV, Nespryadko SV, Vinnitska AB, Usenko VS, Gout IT and Filonenko VV: Immunohistochemical analysis of S6K1 and S6K2 expression in endometrial adenocarcinomas. Exp Oncol 26: 287-293, 2004.

18. Pardo OE, Wellbrock C, Khanzada UK, Aubert M, Arozarena I, Davidson S, Bowen F, Parker PJ, Filonenko VV, Gout IT, et al: FGF-2 protects small cell lung cancer cells from apoptosis through a complex involving PKCepsilon, B-Raf and S6K2. EMBO J 25: 3078-3088, 2006.
19. Roy R, Durie D, Li H, Liu BQ, Skehel JM, Mauri F, Cuorvo LV, Barbareschi M, Guo L, Holcik M, et al: hnRNPA1 couples nuclear export and translation of specific mRNAs downstream of FGF-2/S6K2 signalling. Nucleic Acids Res 42: 12483-12497, 2014.

20. Datta SR, Dudek H, Tao X, Masters S, Fu H, Gotoh Y and Greenberg ME: Akt phosphorylation of BAD couples survival signals to the cell-intrinsic death machinery. Cell 91: 231-241, 1997.

21. Sun Y, He W, Luo M, Zhou Y, Chang G, Ren W, Wu K, Li X, Shen J, Zhao X and Hu Y: SREBP1 regulates tumorigenesis and prognosis of pancreatic cancer through targeting lipid metabolism. Tumour Biol 36: 4133-4141, 2015.

22. Livak KJ and Schmittgen TD: Analysis of relative gene expression data using real-time quantitative PCR and the 2(-Delta Delta C(T)) method. Methods 25: 402-408, 2001.

23. Sun Y, Zhao X, Yao Y, Qi X, Yuan Y and Hu Y: Connexin 43 interacts with Bax to regulate apoptosis of pancreatic cancer through a gap junction-independent pathway. Int J Oncol 41: 941-948, 2012 .

24. Sun Y, Zhao X, Luo M, Zhou Y, Ren W, Wu K, Li X, Shen J and $\mathrm{Hu}$ Y: The pro-apoptotic role of the regulatory feedback loop between miR-124 and PKM1/HNF4alpha in colorectal cancer cells. Int J Mol Sci 15: 4318-4332, 2014.

25. Wang Z, Jeon HY, Rigo F, Bennett CF and Krainer AR Manipulation of PK-M mutually exclusive alternative splicing by antisense oligonucleotides. Open Biol 2: 120133, 2012.

26. Anastasiou D, Yu Y, Israelsen WJ, Jiang JK, Boxer MB, Hong BS, Tempel W, Dimov S, Shen M, Jha A, et al: Pyruvate kinase M2 activators promote tetramer formation and suppress tumorigenesis. Nat Chem Biol 8: 839-847, 2012.

27. Global Burden of Disease Cancer Collaboration, Fitzmaurice $C$, Dicker D, Pain A, Hamavid H, Moradi-Lakeh M, MacIntyre MF, Allen C, Hansen G, Woodbrook R, et al: The Global Burden of Cancer 2013. JAMA Oncol 1: 505-527, 2015.

28. DeBerardinis RJ, Lum JJ, Hatzivassiliou G and Thompson CB: The biology of cancer: Metabolic reprogramming fuels cell growth and proliferation. Cell Metab 7: 11-20,2008.

29. Fang S and Fang X: Advances in glucose metabolism research in colorectal cancer. Biomed Rep 5: 289-295, 2016.

30. Vellinga TT, Borovski T, de Boer VC, Fatrai S, van Schelven S, Trumpi K, Verheem A, Snoeren N, Emmink BL, Koster J, et al: SIRT1/PGC1 $\alpha$-dependent increase in oxidative phosphorylation supports chemotherapy resistance of colon cancer. Clin Cancer Res 21: 2870-2879, 2015.

31. Ward PS and Thompson CB: Metabolic reprogramming: A cancer hallmark even Warburg did not anticipate. Cancer cell 21: 297-308, 2012.

32. Hirschey MD, DeBerardinis RJ, Diehl AME, Drew JE, Frezza C, Green MF, Jones LW, Ko YH, Le A, Lea MA, et al: Dysregulated metabolism contributes to oncogenesis. Semin Cancer Biol 35 (Suppl): S129-S150, 2015.

33. Zhou CF, Li XB, Sun H, Zhang B, Han YS, Jiang Y, Zhuang QL, Fang $\mathrm{J}$ and $\mathrm{Wu} \mathrm{GH}$ : Pyruvate kinase type M2 is upregulated in colorectal cancer and promotes proliferation and migration of colon cancer cells. IUBMB Life 64: 775-782, 2012.

34. Wong N, De Melo J and Tang D: PKM2, a central point of regulation in cancer metabolism. Int J Cell Biol 2013: 242513, 2013.

35. Jean-Philippe J, Paz S and Caputi M: hnRNP A1: The Swiss army knife of gene expression. Int J Mol Sci 14: 18999-19024, 2013.

36. Goina E, Skoko N and Pagani F: Binding of DAZAP1 and hnRNPA1/A2 to an exonic splicing silencer in a natural BRCA1 exon 18 mutant. Mol Cell Biol 28: 3850-3860, 2008. 